\title{
Transient ischemic dilation: An old but not obsolete marker of extensive coronary artery disease
}

\author{
Tali Sharir, $M D^{\mathrm{a}, \mathrm{b}}$ \\ a Department of Nuclear Cardiology, Assuta Medical Center, Tel Aviv, Israel \\ b Faculty of Health Sciences, Ben Gurion University of the Negev, Beer Sheba, Israel
}

Received Sep 4, 2017; accepted Sep 5, 2017

doi: $10.1007 /$ s12350-017-1082-x

\section{See related article, pp. 724-737}

Myocardial perfusion imaging (MPI) has been used in the detection and evaluation of ischemic heart disease for the past 40 years. Image interpretation is based on the identification of reversible and fixed perfusion deficit. However, several adjunctive markers have been suggested over the years to increase the diagnostic and prognostic value of the test, and improve the detection of extensive coronary artery disease (CAD). One of these ancillary markers has been referred to as transient ischemic dilation (TID) of the left ventricle (LV), which is detected as increased ventricular cavity size at stress compared to rest. The concept of TID was initially described by Stolzenberg in $1980^{1}$ on planar stress-redistribution thallium scans, and further established by Weiss et al. in 1987 as a highly specific marker of severe multivessel CAD. ${ }^{2}$ The pathophysiologic mechanism responsible for this phenomenon is probably comprised of several contributors. A generally accepted explanation is global subendocardial ischemia at stress. ${ }^{3,4}$ In the presence of extensive CAD, epicardial perfusion pressure is reduced at stress, whereas left ventricular enddiastolic pressure is elevated, resulting in subendocardial hypoperfusion and ischemia. At this condition, the uptake of radiotracer in the subendocardial region is markedly reduced, resulting in a thinner appearance of the LV wall and larger inner ventricular cavity

Reprint requests: Tali Sharir, MD, Department of Nuclear Cardiology, Assuta Medical Center, 96 Igal Alon, C Building, 67891 Tel Aviv, Israel; tsharir@gmail.com

J Nucl Cardiol 2018;25:738-41.

1071-3581/\$34.00

Copyright (C) 2017 American Society of Nuclear Cardiology. compared to baseline condition. Using SPECT thalliumredistribution, Iskandrian et al. $^{3}$ demonstrated thinner ventricular wall and larger endocardial size at adenosine stress compared to redistribution images among $30 \%$ of patients with CAD, whereas only $6 \%$ of the patients had true ventricular dilation with increased epicardial volume.

Another mechanism which has been suggested to explain TID is post-stress stunning due to stress induced ischemia. ${ }^{5,6}$ According to this explanation, post-stress stunning results in end-systolic volume enlargement of the left ventricle. Since non-gated images used for TID calculation reflect summation of counts originating from the whole cardiac cycle, reduced post-stress ventricular contraction with larger end-systolic volume results in increased non-gated LV volume and increased TID ratio. Betesti et al. ${ }^{5}$ demonstrated that post-stress $\mathrm{LV}$ dilation by gated SPECT reflected endocardial post-ischemic stunning with reduced ejection fraction and thickening score, and was associated with more severe clinical, angiographic, and perfusion parameters. Another investigation supporting this mechanism demonstrated LV dysfunction at stress echocardiography among patients with TID at MPI. ${ }^{6}$ True LV dilation at stress with epicardial volume enlargement is apparently uncommon, and less likely to explain the occurrence of TID.

In the current issue of the journal, Alama et al. from Toronto, Canada, conducted a meta-analysis on the diagnostic performance of TID in the detection of severe and extensive CAD. The final quantitative meta-analysis was based on 13 studies comprising a total of 2037 patients, which evaluated the diagnostic value of TID, and reported results of coronary angiography, either invasive or CT-based. Definition of severe and extensive CAD varied within the studies, but most of them included severe angiographic proximal left anterior descending diameter stenosis of $\geq 90 \%$ in their 
definition, and additional severe stenosis of any vessel, or any severe stenosis of two or three coronary arteries. Moderate-severe left main stenosis $\geq 70 \%$ was also included in the definition of several studies. Only two studies were based on thallim-201 and used planar images for manual calculation of TID. Of the rest, most utilized Tc-99m, and only few were based on dual-isotope rest Tl-201/ stress Tc-99m or PET rubidium-82, all utilizing quantitative TID assessment. Most of the studies utilized quantitative software for objective calculation of TID. As expected, pooled data analysis demonstrated low sensitivity of $44 \%$ with a wide range of individual study sensitivity of $7 \%$ to $91 \%$, and high specificity of $88 \%$, ranging from $62 \%$ to $96 \%$. Importantly, the threshold of TID ratio defining high risk of severe CAD varied from 1.12 to 1.38 within the studies, without distinct characteristics with respect to the isotope used, whether thallium-201 or technetium-99m. Interestingly, subgroup analysis based on the type of stress performed yielded higher diagnostic value with larger area under the ROC curve for exercise vs vasodilator stress $(0.92$ vs 0.78 , respectively, $p<0.001)$. A possible explanation for this observation might be a more frequent occurrence of true ischemia and post-stress stunning with LV end-systolic volume enlargement associated with exercise compared to vasodilator stress, but this mechanism has not been evaluated.

A chronological assessment of the studies included in the current meta-analysis reveals the evolution in the field of nuclear cardiology over the past four decades, which occurred in several domains (Figure 1). ${ }^{2,7-13}$ The exclusive use of thallium-201 has been replaced by Tc$99 \mathrm{~m}$ labeled radiotracers and dual-isotope protocols. Planar imaging systems were developed into SPECT systems, and SPECT systems were advanced from single head to dual-detector gamma cameras. Lately, solid state CZT systems emerged in clinical practice. ${ }^{14}$ PET rubidium-82 MPI has been introduced as an alternative to SPECT MPI. ${ }^{11}$ In addition, quantitative analysis of perfusion and function parameters has gained an important role, and became an integral part of MPI interpretation. The assessment of TID ratio has been changed in parallel to these global advances in nuclear cardiology: from thallium-201 to Tc-99m based radiotracers, from planar imaging to SPECT and PET technologies, and from manual area calculation to fully automatic 3-D assessment of LV chamber volume. Yet, TID remained a robust and important marker of highrisk scan, highly specific for severe multivessel CAD, across all imaging techniques, type of radio-isotopes and protocols used, and type of stress performed. It should be noted that although gated SPECT became the standard acquisition mode in MPI, and TID ratio could be derived from either gated end-diastolic or end-systolic stress-rest ratio, the non-gated TID ratio remained the clinically adjunctive parameter for prediction of extensive CAD. $\mathrm{Xu}$ et al. ${ }^{12}$ demonstrated that the non-gated TID ratio identified severe CAD more efficiently than either end-diastolic or end-systolic TID ratios.

The current meta-analysis evaluated the TID ratio as an isolated parameter, without consideration of perfusion findings. However, in the clinical setting, TID is used as an adjunctive marker to perfusion findings, supporting the diagnosis of obstructive CAD, and assisting in identification of extensive disease. Using a CZT camera, Jameria el al. ${ }^{14}$ demonstrated that TID ratio could be used as adjunctive marker to perfusion deficit, and is most effective among patients with ejection fraction $\geq 50 \%$. Xu et al. ${ }^{12}$ demonstrated added diagnostic value of TID over perfusion alone. They show that TID ratio was more frequently elevated among patients with moderate-severe perfusion abnormality (total perfusion deficit $\geq 10 \%$ ) compared to patients with smaller perfusion deficit $(<10 \%)$. Moreover, the combination of either severe perfusion deficit ( $\geq 10 \%$ ) or mild perfusion deficit $(3 \%-10 \%)+$ elevated TID ratio $(\geq 1.19)$ was associated with higher sensitivity for extensive CAD compared to perfusion deficit $\geq 10 \%$ alone. Thus, increased TID ratio associated with mild perfusion deficit might suggest more extensive CAD than observed by perfusion findings. Data on the diagnostic value of abnormal TID ratio in an otherwise normal MPI is controversial. A recent study by Halligan et al. ${ }^{15}$ evaluated 1553 patients who underwent both SPECT MPI and cardiac CT within 1 month. They compared 20 TIDpositive patients and 48 TID-negative matched patient population, and found no significant difference in the presence and extent of atherosclerosis, the degree of coronary artery stenosis, or the calcium score at cardiac CT. In contrast, Nakanishi et al. ${ }^{16}$ found that abnormal TID ratio was a significant predictor of extensive CAD among patients with normal MPI who underwent invasive coronary angiography within 60 days.

The current study by Alama et al. also presents the results of systematic review on the prognostic value of TID. Unanimously, all eight studies included in the review demonstrated worse outcome among patients with abnormal compared to normal TID. Importantly, abnormal TID predicted higher event rate even among patients with normal or mildly abnormal perfusion, particularly among patients with diabetes mellitus. ${ }^{17}$

In addition to TID, several imaging findings have been used as markers of extensive CAD. During the early 1980's increased post-stress lung uptake of thallium-201 on MPI has been shown to correlate with severe CAD and left ventricular dysfunction. ${ }^{18}$ However, the abandon of thallium-201 and shift to Tc-99m radiotracers have put 


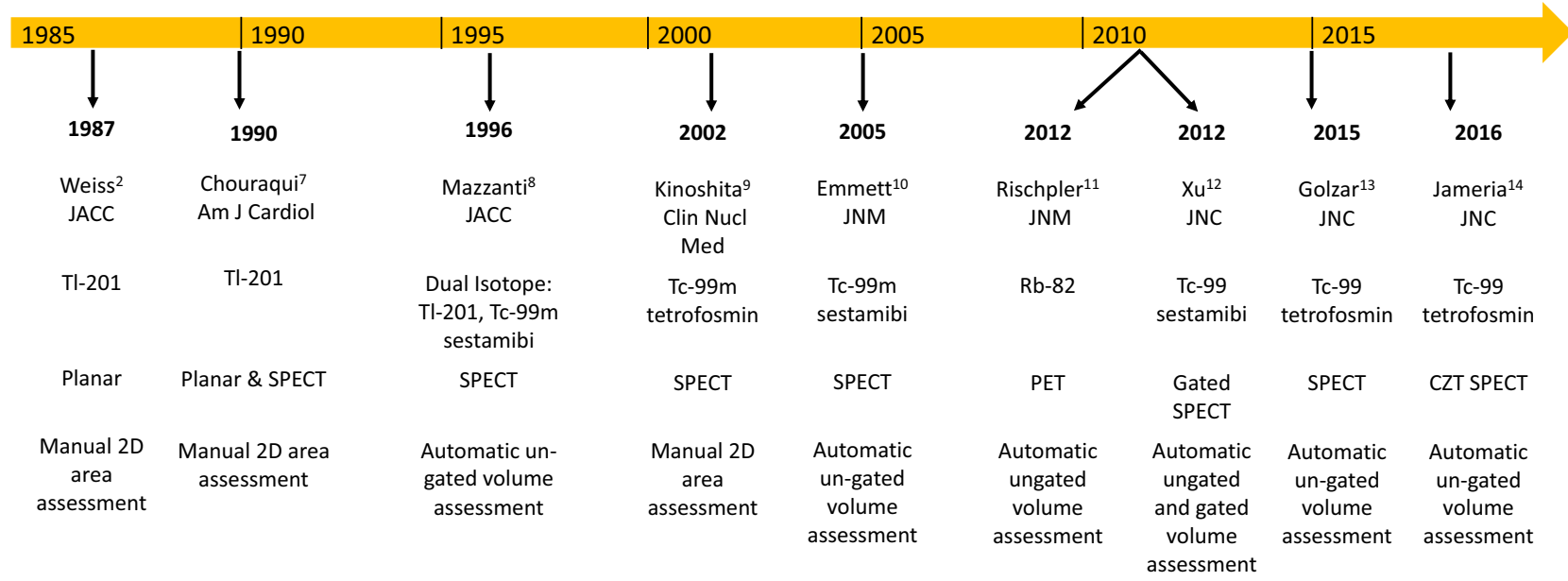

Figure 1. Timeline of articles assessing the diagnostic value of TID, indicating isotope used, imaging technology, and method of TID ratio assessment.

the lid on this previously helpful marker. Post-stress stunning with a decrease in ejection fraction and the appearance of new regional wall motion abnormality following exercise or pharmacologic stress has been shown as an important marker of severe CAD. ${ }^{19,20}$ In recent years, myocardial blood flow and flow reserve by positron emission tomography have been used to identify the extent of CAD. Using rubidium-82 positron emission tomography, Ziadi et al. demonstrated that myocardial flow reserve $<2$ provided added value to relative perfusion in predicting three vessel CAD, incremental over the summed stress score. CZT SPECT systems have also been used for assessment of coronary flow reserve and the extent of CAD. ${ }^{21}$ Coronary artery calcium scoring is an important measure of the extent of atherosclerosis. Combining coronary calcium with MPI may improve identification of obstructive CAD. Several studies have demonstrated that very high calcium score $(>1000)$ is frequently associated with more extensive obstructive CAD than seen by MPI. ${ }^{22,23}$ These measures, whether based on MPI itself like the TID, or require special technologies and expertise, improve the detection of high-risk obstructive $\mathrm{CAD}$, and should be incorporated into nuclear testing and interpretation according to their availability.

\section{Disclosure}

Tali Sharir has no conflict of interest.

\section{References}

1. Stolzenberg J. Dilatation of left ventricular cavity on stress thallium scan as an indicator of ischemic disease. Clin Nucl Med 1980;5:289-91.
2. Weiss AT, Berman DS, Lew AS, et al. Transient ischemic dilation of the left ventricle on stress thallium-201 scintigraphy: A marker of severe and extensive coronary artery disease. J Am Coll Cardiol 1987;9:752-9.

3. Iskandrian AS, Heo J, Nguyen T, Lyons E, Paugh E. Left ventricular dilatation and pulmonary thallium uptake after singlephoton emission computer tomography using thallium-201 during adenosine-induced coronary hyperemia. Am J Cardiol 1990;66:801-11.

4. Takeishi Y, Tono-oka I, Ikeda K, Komatani A, Tsuiki K, Yasui S. Dilatation of the left ventricular cavity on dipyridamole thallium201 imaging: A new marker of triple-vessel disease. Am Heart J 1991;121:466-75.

5. Bestetti A, Di Leo C, Alessi A, Triulzi A, Tagliabue L, Tarolo GL. Post-stress end-systolic left ventricular dilation: A marker of endocardial post-ischemic stunning. Nucl Med Commun 2001;22:685-93.

6. Slutsky R, Karliner J, Ricci D, et al. Response of left ventricular volume to exercise in man assessed by radionuclide equilibrium angiography. Circulation 1979;60:565-71.

7. Chouraqui P, Rodrigues EA, Berman DS, Maddahi J. Significance of dipyridamole-induced transient dilation of the left ventricle during thallium-201 scintigraphy in suspected coronary artery disease. Am J Cardiol 1990;66:689-94.

8. Mazzanti M, Germano G, Kiat H, et al. Identification of severe and extensive coronary artery disease by automatic measurement of transient ischemic dilation of the left ventricle in dual-isotope myocardial perfusion SPECT. Am Coll Cardiol 1996;27:1612-20.

9. Kinoshita N, Sugihara H, Adachi Y, et al. Assessment of transient left ventricular dilatation on rest and exercise on Tc-99m tetrofosmin myocardial SPECT. Clin Nucl Med 2002;27:34-9.

10. Emmett L, Van Gaal WJ, Magee M, et al. Prospective evaluation of the impact of diabetes and left ventricular hypertrophy on the relationship between ischemia and transient ischemic dilation of the left ventricle on single-day adenosine Tc-99m myocardial perfusion imaging. J Nucl Cardiol 2008;15:638-43.

11. Rischpler C, Higuchi T, Fukushima K, et al. Transient ischemic dilation ratio in 82Rb PET myocardial perfusion imaging: Normal values and significance as a diagnostic and prognostic marker. $\mathbf{J}$ Nucl Med 2012;53:723-30.

12. Xu Y, Arsanjani R, Clond M, et al. Transient ischemic dilation for coronary artery disease in quantitative analysis of same-day 
sestamibi myocardial perfusion SPECT. J Nucl Cardiol 2012;19:465-73.

13. Golzar Y, Olusanya A, Pe N, et al. The significance of automatically measured transient ischemic dilation in identifying severe and extensive coronary artery disease in regadenoson, single-isotope technetium-99m myocardial perfusion SPECT. J Nucl Cardiol 2015;22:526-34.

14. Jameria ZA, Abdallah M, Dwivedi A, et al. Computer derived transient ischemic dilation ratio for identifying extensive coronary artery disease using a CZT camera and imaging in the upright position. J Nucl Cardiol 2016. doi:10.1007/s12350-016-0515-2.

15. Halligan WT, Morris PB, Schoepf UJ, et al. Transient ischemic dilation of the left ventricle on SPECT: Correlation with findings at coronary CT angiography. J Nucl Med 2014;55:917-22.

16. Nakanishi R, Gransar H, Slomka P, et al. Predictors of high-risk coronary artery disease in subjects with normal SPECT myocardial perfusion imaging. Nucl Cardiol 2016;23:530-41.

17. Doukky R, Frogge N, Bayissa YA, et al. The prognostic value of transient ischemic dilatation with otherwise normal SPECT myocardial perfusion imaging: A cautionary note in patients with diabetes and coronary artery disease. J Nucl Cardiol 2013;20:77484.

18. Kushner FG, Okada RD, Kirshenbaum HD, Boucher CA, Strauss HW, Pohost GM. Lung thallium-201 uptake after stress testing in patients with coronary artery disease. Circulation 1981;63:341-7.
19. Sharir T, Bacher-Stier C, Dhar S, et al. Identification of severe and extensive coronary artery disease by postexercise regional wall motion abnormalities in Tc-99m sestamibi gated single-photon emission computed tomography. Am J Cardiol 2000;86:1171-5.

20. Emmett L, Iwanochko RM, Freeman MR, Barolet A, Lee DS, Husain M. Reversible regional wall motion abnormalities on exercise technetium-99m-gated cardiac single photon emission computed tomography predict high-grade angiographic stenosis. J Am Coll Cardiol 2002;39:991-8.

21. Ziadi MC, Dekemp RA, Williams K, et al. Does quantification of myocardial flow reserve using rubidium-82 positron emission tomography facilitate detection of multivessel coronary artery disease? J Nucl Cardiol 2012;19:670-80.

22. Yuoness SA, Goha AM, Romsa JG, et al. Very high coronary artery calcium score with normal myocardial perfusion SPECT imaging is associated with a moderate incidence of severe coronary artery disease. Eur J Nucl Med Mol Imaging 2015;42:154250.

23. Brodov Y, Gransar H, Dey D, et al. Combined quantitative assessment of myocardial perfusion and coronary artery calcium score by hybrid $82 \mathrm{Rb}$ PET/CT improves detection of coronary artery disease. J Nucl Med 2015;56:1345-50. 